\title{
The Mass Distribution in the Nearby Universe
}

\author{
Luiz N. da Costa \\ European Southern Observatory, Karl-Schwarzschild-Str. 2, D-85748 Garching b. \\ München, Germany \\ Observatório Nacional, Rua Gen. José Cristino 77, São Cristovão, Rio de Janeiro, Brazil \\ Wolfram Freudling \\ Space Telescope-European Coordinating Facility and European Southern Observatory, \\ Karl-Schwarzschild-Str. 2, D-85748 Garching b. München, Germany \\ Gary Wegner \\ Dept. of Physics and Astronomy, Dartmouth College, Hanover, NH 03755 \\ Riccardo Giovanelli, Martha P. Haynes \\ Center for Radiophysics and Space Research and National Astronomy and Ionosphere \\ Center $^{1}$, Cornell University, Ithaca, NY 14853 \\ John J. Salzer \\ Dept. of Astronomy, Wesleyan University, Middletown, CT 06457
}

\footnotetext{
${ }^{1}$ The National Astronomy and Ionosphere Center is operated by Cornell University under a cooperative agreement with the National Science Foundation.
} 
Received _ ; accepted _ 


\begin{abstract}
We present a new reconstruction of the mass density and the peculiar velocity fields in the nearby universe using recent measurements of Tully-Fisher distances for a sample of late spirals. We find significant differences between our reconstructed fields and those obtained in earlier work: overdensities tend to be more compact while underdense regions, consisting of individual voids, are more abundant. Our results suggest that voids observed in redshift surveys of galaxies represent real voids in the underlying matter distribution. While we detect a bulk velocity of $\sim 300 \mathrm{~km} \mathrm{~s}^{-1}$, within a top-hat window $6000 \mathrm{~km} \mathrm{~s}^{-1}$ in radius, the flow is less coherent than previously claimed, exhibiting a bifurcation towards the Perseus-Pisces and the Great Attractor complexes. This is the first time that this feature is seen from peculiar velocity measurements. The observed velocity field resembles, more closely than any previous reconstruction, the velocity field predicted from self-consistent reconstructions based on all-sky redshift surveys. This better match is likely to affect estimates of the parameter $\beta=\Omega^{0.6} / b$ and its uncertainty based on velocity-velocity comparisons.
\end{abstract}

Subject headings: galaxies: distances and redshifts; photometry - cosmology: observations; cosmic microwave background 


\section{Introduction}

Fluctuations in the mass distribution on intermediate scales can be probed by peculiar velocity measurements, by measurements of anisotropies in the cosmic background radiation at the $1^{\circ}$ scale and by distortions of galaxy images induced by weak field lensing. Despite several pitfalls, the peculiar motion approach still remains the most direct and the easiest to interpret, provided that large-scale structures grow gravitationally and fluctuations in the mass distribution induce the observed peculiar velocities of galaxies. Mapping the peculiar velocity field can thus provide information useful to constrain the mass power-spectrum, the relation between the galaxy and matter distributions, and the value of the cosmological density parameter $\Omega$ on large scales.

In recent years, considerable effort has been made to understand the nature of the peculiar velocity field and the determination of the parameter $\beta=\Omega^{0.6} / b$, where $b$ is the linear biasing parameter. Measurements of $\beta$ have been attempted either from the comparison of the observed fluctuations in the galaxy distribution with those obtained from dynamical studies or from the direct comparison of the measured peculiar velocity field to that predicted from dynamically self-consistent reconstruction of the density field from all-sky redshift surveys (for a review see Dekel 1994). These estimates based on peculiar velocity measurements have generally led to higher values than those obtained on small scales by other methods (e.g. Strauss \& Willick 1995). The reason for this inconsistency remains an open question.

Until recently, most of the work was based on compilations of distance measurements by different authors which were sparse, inhomogeneous and probed a shallow volume in a non-uniform way (Bertschinger et al. 1990, Dekel 1994). Although several new peculiar velocity surveys have been carried out (Willick 1990, Courteau et al. 1993, Mathewson, Ford \& Buchhorn 1992b, Wegner et al. 1996), none covers the whole sky uniformly. Efforts 
to homogenize the existing datasets are currently underway (Willick et al. 1996) but error estimates for the distances of individual galaxies are likely to continue to be uncertain. This directly affects the estimation of the bias which might, in the case of the inhomogeneous Malmquist bias correction, lead to spurious detection of density fluctuations.

The need for a sample with uniform sky coverage and homogeneous measurements has prompted us to carry out an independent redshift-distance survey of Sbc-Sc galaxies, based on I-band Tully-Fisher (TF) distances, covering essentially the whole sky $\left(\mathrm{dec} \geq-45^{\circ}\right.$ and $|b| \geq 10^{\circ}$ ). The sample has well-defined selection criteria and new measurements were carried out in order to assure the homogeneity of the data. This data set was complemented with galaxies drawn from Mathewson, Ford \& Buchhorn (1992b, MFB), appropriately converted to our magnitude and width scale, yielding a combined sample of about 1300 field galaxies (hereafter SFI sample) and 500 galaxies in clusters. Details on sample selection and the procedure adopted to combine the two datasets can be found in Giovanelli et al. (1994, 1995a,b). Here, we present a summary of the results of a reconstruction of the three-dimensional velocity and density fields obtained from the SFI sample. We use the 3D reconstruction method primarily to illustrate the nature of the sample as compared to those currently available. A more quantitative analysis will be the subject of future papers. In section 2, we briefly describe the reconstruction method and discuss the approach used to correct for the various biases that affect the distance estimates. Our main results are presented in section 3, while in section 4 we summarize our conclusions.

\section{Analysis of the SFI Sample}

Distances of galaxies were estimated using the direct I-band TF relation derived from the combination of data for 24 clusters (Giovanelli et al. 1996 a,b). However, errors in the distance measurements lead to biases which directly affect the peculiar velocity field and thus 
the derived density field. The biases result from the coupling of uncertainties in the distance estimate with inhomogeneities in the galaxy distribution, and the selection of the sample. The latter is particularly important for the Sc sample because the adopted redshift-dependent selection criteria (e.g. Giovanelli et al. 1994) implies that near each redshift limit outflowing galaxies are preferentially excluded from the sample. This effect becomes dominant at large distances leading to a spurious systematic infall of galaxies at the outer edge of the surveyed volume. In order to deal with this problem we have resorted to a numerical approach to estimate the bias field at each point in estimated distance space (Freudling et al. 1995). The method, which is based on mock catalogs with characteristics similar to the data, allows for the simultaneous correction of the homogeneous and inhomogeneous Malmquist bias, and biases introduced by selection effects. The mock catalogs are generated from the distribution of optically-selected galaxies in real space. This distribution was obtained using an iterative method of reconstructing a dynamically self-consistent distribution of galaxies from all-sky redshift surveys (Optical-IRAS sample) using a quasi-linear theory (Freudling, da Costa \& Pellegrini 1994).

The bias correction is sensitive to the value adopted for the scatter in distance estimates. Attempts to use the scatter as determined for the TF relation of galaxies in clusters led to an overcorrection of the velocity field. In order to overcome this problem we have also used the mock catalogs to determine the scatter in the distance estimates of field galaxies, taking advantage of the homogeneity of the sample. This was done by comparing the observed radial peculiar velocities at large distances with those of mock catalogs, since these velocities are primarily produced by biases which depend on the assumed scatter. The scatter of the mock samples was adjusted so that the radial variation of the peculiar velocities at large distances in the mock catalog match the observations. The derived scatter of $\lesssim 0.4$ mag depends on the HI line-width, and is in any case smaller than that obtained for galaxies in clusters (Giovanelli et al. 1996a). A more extensive discussion of the motivation and the 
details for the adopted procedure will be presented in da Costa et al. (1996).

Distances were calculated from the TF relation and the radial component of the peculiar velocity $u=c z_{C M B}-d_{c}$, where $d_{c}$ is the "fully" corrected distance estimate. Galaxies in clusters were assigned to a single object. A small fraction of galaxies $(\sim 6 \%)$ with small line widths $(\log w<2.25)$ were discarded because of the large fractional errors in the measurement of their widths. The final input sample used in the reconstruction of the density field consists of 1234 independent points.

The computed distances and peculiar velocities are the input data to reconstruct the density and three-dimensional peculiar velocity fields. For the reconstruction, we use the standard assumption of irrotational flow which allows calculation of the scalar velocity potential field from the integral along radial paths of the line-of-sight component of the peculiar velocity. Our algorithm is similar to that discussed by Dekel, Bertschinger \& Faber (1990). We adopt a grid in spherical coordinates of 20 equal shells out to a maximum radius of 7500 $\mathrm{km} \mathrm{s}^{-1}$ and 64 latitude and longitude circles. The measured radial peculiar velocities were smoothed using a Gaussian tensor window with a fixed smoothing scale of $R_{w}=900 \mathrm{~km} \mathrm{~s}^{-1}$. Our methodology was extensively tested using mock catalogs with the same number of objects and selection criteria as the data, which were also used to determine the uncertainties in the reconstruction (da Costa et al. 1996).

\section{Results}

The reconstructed density fluctuation field $\delta$ along the Supergalactic plane on a cubic grid of spacing $500 \mathrm{~km} \mathrm{~s}^{-1}$ for a fully corrected model is displayed in figure 1, where we show in the top plane density contours in $\delta=0.2$ intervals, in the middle plane surface density maps with the contour level proportional to the density contrast $\delta$ and in the bottom plane 
density contrast maps. We use a Cartesian supergalactic coordinate system with SGX-axis pointing to $L=0^{\circ}$ and the SGY-axis pointing nearly to the North Galactic Pole.

¿From a visual inspection of the map one can recognize some well-known structures in the nearby volume such as the Great Attractor (GA, SGX $\sim-2000 \mathrm{~km} \mathrm{~s}^{-1}$, SGY $500 \mathrm{~km} \mathrm{~s}^{-1}$ ), the Perseus-Pisces (PP) complex (SGX $\sim 6000 \mathrm{~km} \mathrm{~s}^{-1}, \mathrm{SGY} \sim-1000 \mathrm{~km} \mathrm{~s}^{-1}$ ) and the large void $\left(\mathrm{SGX} \sim 2500 \mathrm{~km} \mathrm{~s}^{-1}\right.$, SGY $\sim 0 \mathrm{~km} \mathrm{~s}^{-1}$ ) between the GA and the PP. We can also see traces of the Coma/A1367 supercluster along SGY $\sim 7000 \mathrm{~km} \mathrm{~s}^{-1}$, and the Cetus region ( $\mathrm{SGX} \sim 500 \mathrm{~km} \mathrm{~s}^{-1}$, SGY $\sim-6000 \mathrm{~km} \mathrm{~s}^{-1}$ ). These structures are located roughly at the same position as in the $I R A S$ reconstructed density field (e.g. Strauss \& Willick 1995)

In comparison to earlier work (e.g. Dekel 1994), better sampling of the northern hemisphere apparently leads to significant differences in the density field especially in the region not covered by MFB. Some important differences can be seen: 1) in the foreground of the Coma/A1367 supercluster, where now a large void is detected; 2) in the PP region, which appears as a compact high density peak; and 3) in the Great Attractor region, which looks less prominent. Examination of the reconstructed density field in three-dimensions also suggests that the GA consists of different sub-structures, much in the way seen in redshift surveys (e.g. Willmer et al. 1995).

A striking feature of our reconstructed density field is the existence of several voids surrounded by tenuous structures. Among them is the nearby void separated from the voids in the foreground of the Coma/A1367 supercluster by a small amplitude coherent structure, which we identify as the Local Supercluster. It is, as expected, much less prominent than other structures within the volume. Other prominent voids are the void behind the GA $\left(\mathrm{SGX} \sim-6500 \mathrm{~km} \mathrm{~s}^{-1}, \mathrm{SGY} \sim 1500 \mathrm{~km} \mathrm{~s}^{-1}\right.$ ) and the Sculptor void (SGX $\sim-2000 \mathrm{~km} \mathrm{~s}^{-1}$, $\mathrm{SGY} \sim-6000 \mathrm{~km} \mathrm{~s}^{-1}$ ). Both voids have been detected in galaxy redshift maps (e.g. da 
Costa et al. 1988, Geller \& Huchra 1989) but are now seen in the mass distribution with density contrasts reaching $\delta \lesssim-0.6$, as indicated by the contours in the top plane.

Several other positive and negative fluctuations are seen near the outer edge of our survey volume. Comparison with the Optical-IRAS and IRAS density fields indicate that most are likely to be real structures. However, it is important to emphasize that the error in the density field derived from mock catalogs, which is smaller than 0.2 within $5000 \mathrm{~km} \mathrm{~s}^{-1}$, grows rapidly with radius. At $6000 \mathrm{~km} \mathrm{~s}^{-1}$ the error is estimated to be about 0.4 , corresponding to two contour levels. Therefore, the amplitude and location of structures beyond $5000 \mathrm{~km} \mathrm{~s}^{-1}$ are very poorly determined. This is also true for the Coma/A1367 and PP superclusters. However, although PP itself is poorly mapped, its influence in the velocity field extends over a large volume and into the region where the errors are small and the results insensitive to the details of the inhomogeneous bias correction.

The density field reconstructed with the SFI sample is characterized by overdensities which are compact and underdensities which are large, have regular shape and have a high density contrast. This is in marked contrast to earlier reconstructions. Our results suggest the existence of real voids in the matter distribution, qualitatively similar to those observed in galaxy redshift surveys (e.g. da Costa et al. 1994). This supports the contention that galaxies delineate real voids in the underlying matter distribution which may impose severe constraints in the amplitude of the mass power-spectrum (e.g. Piran et al. 1993).

In Figure 2, we show the velocity field along the Supergalactic plane superimposed to the density field shown in figure 1. This figure should be compared to a similar map presented by Dekel (1994). In contrast to Dekel's result our velocity field is qualitatively similar to the predicted IRAS velocity field (e.g. Yahil 1988) showing a region where the flow bifurcates towards the GA and towards the PP complex. If true, this resolves a longstanding discrepancy between predicted and measured velocity fields. Moreover, an infall into PP is 
clearly observed while none is seen in Dekel's (1994) reconstruction. This contrasts with the interpretation of Willick (1990) who claims that the principle feature of the velocity field in the PP region is a coherent streaming towards the Local Group'. Willick's smaller sky coverage of the PP region could well be the cause of this difference.

On the opposite side of the figure, we find that the GA is less important than originally believed. Instead of being responsible for most of the motion in the nearby volume, there is strong evidence that it is smaller and less massive than originally estimated by Lynden-Bell et al. (1988). This is probably due to the larger surveyed volume and better sampling of the PP region. Note, in particular, that the amplitude of the backflow into the GA is small. Instead, the flow field in the upper left hand side of Figure 2 could be explained by a mass concentration beyond the survey volume pulling the GA. This would explain the lack of an unambiguous signature for backside infall in the survey of Burstein, Faber \& Dressler (1990). Finally, the volume-weighted bulk velocity as measured within a top-hat window $6000 \mathrm{~km} \mathrm{~s}^{-1}$ in radius is about $300 \mathrm{~km} \mathrm{~s}^{-1}$ in the direction $L \sim 160^{\circ}$ and $B \sim-20^{\circ}$, where $L$ and $B$ are the supergalactic coordinates. These values are very close to those reported by Dekel (1994). However, the flow shows an overall shear unlike the coherence suggested by Mathewson, Ford and Buchhorn (1992a) and Courteau et al. (1993).

There are several possible explanations for the observed differences between the results of our reconstruction and those of earlier work (e.g. Dekel 1994). The uniform sampling over the sky and depth is certainly a major factor, as pointed out above. Differences in the assumed scatter properties and methods for inhomogeneous Malmquist bias correction could also contribute to the position-dependent differences observed. Differences in the relative zero-points between different data sets in the sample used by Dekel (1994) could be another reason for the observed discrepancies. A detailed comparison of the two datasets will be required to better understand the reason for the discrepancies. 


\section{Conclusions}

The picture that emerges from our reconstruction brings together the somewhat fragmentary view previously held. The main results may be summarized as follows:

1- The positive fluctuations of the matter density field tend to be more compact while well-defined voids are more abundant than those obtained in previous reconstructions.

2- Comparison with redshift maps suggests that galaxies delineate real voids in the mass distribution. These voids tend to be separated by relative low-density structures which correspond to the filamentary and wall-like structures observed in the galaxy distribution.

3- The GA is significantly less prominent than previously claimed. There is also some indication for sub-structures.

4- An outside mass concentration may be pulling the GA. This induced motion overshadows the backflow and explains the lack of convincing evidence of such a flow.

5- The PP supercluster makes a significant contribution to the flow in its neighborhood leading to an infall into the supercluster seen for the first time from peculiar velocity measurements.

6- The measured mean bulk velocity within a top-hat window of $6000 \mathrm{~km} \mathrm{~s}^{-1}$ is about

$300 \mathrm{~km} \mathrm{~s}^{-1}$. The flow shows an overall shear which may be consistent with the existence of a mass concentration outside the observed volume.

Our results should affect conclusions drawn earlier regarding the value of $\beta$, or at least the accuracy with which it can be determined, because of the better agreement between the velocity fields reconstructed from peculiar velocity measurements and from the galaxy distribution. The results may also affect the conclusions regarding the biasing of galaxies relative to the mass and the mass power-spectrum. These topics will be pursued in more 
detail in future papers.

We would like to thank A. Dekel for many useful discussions and to the anonymous referee for his constructive comments. $\mathrm{LNdC}$ would like to thank the hospitality of the Institute d'Astrophysique and the Hebrew University where part of this work was carried out. GW thanks for the hospitality of the European Southern Observatory, NSF Grant AST9347714 and the Alexander von Humboldt foundation for partial support. We acknowledge the financial support from U.S. National Science Foundation grants AST94-20505 to RG, AST92-18038 and AST90-23450 to MPH and from the Research Corporation to JJS. The results presented in this paper are based on observations carried out at the European Southern Observatory (ESO), the National Astronomy and Ionosphere Center (NAIC), the National Radio Astronomy Observatory (NRAO), the Kitt Peak National Observatory (KPNO), the Cerro Tololo Interamerican Observatory (CTIO), the Observatory of Paris at Nançay and the Michigan-Dartmouth-MIT Observatory (MDM). NAIC, NRAO, KPNO and CTIO are respectively operated by Cornell University, Associated Universities, inc., and Associated Universities for Research in Astronomy, all under cooperative agreements with the National Science Foundation. 


\section{REFERENCES}

Bertschinger, E., Dekel, A., Faber, S. M., Dressler. A. \& Burstein, D. 1990, ApJ 364, 370.

Courteau, S., Faber, S.M., Dressler, A. and Willick, J.A. 1993, ApJ 412 L51.

Burstein, D., Faber, S. M. \& Dressler, A. ApJ 354, 18

da Costa, L.N., Pellegrini, P.S., Sargent,W.L.W., Tonry, J., Davis, M., Meiksin, A., Latham, L., Menzies, J. \& Coulson, I., 1988, ApJ 327, 544

da Costa, L.N., Geller, M.J., Pellegrini, P.S., Latham, D.W., Fairall, A.P., Marzke, R.O., Willmer, C.N.A., Huchra, J.P., Calderon, J.H., Ramella, M., \& Kurtz, J. 1994, ApJ 424, L1

da Costa et al. 1996, in preparation

Dekel, A., Bertschinger, E. \& Faber, S. M. 1990, ApJ 364, 349

Dekel, A. 1994, ARA\&A 32, 371 in the Universe, ed. V. Rubin \& G. V. Coyne (Princeton: Princeton Univ. Press), 116

Freudling, W., da Costa, L. N. \& Pellegrini, P. S. 1994, MNRAS 268, 943

Freudling, W., da Costa, L. N., Wegner, G., Giovanelli, R, Haynes, M. P. \& Salzer, J.J. 1995, AJ 110,920

Geller, M.G. \& Huchra, J.P. 1989, Science 246, 897

Giovanelli, R., Haynes, M.P., Salzer, J.J., Wegner, G., da Costa, L.N. \& Freudling, W. 1994, AJ 107,2036

Giovanelli, R., Haynes, M.P., Salzer, J.J., Wegner, G., da Costa, L.N. \& Freudling, W. 1995a, AJ 110, 1059

Giovanelli, R., Haynes, M.P., Chamaraux, P., da Costa, L.N., Freudling, W., Salzer, J.J. \& Wegner, G. 1995b, in Examining the Big Bang and Diffuse Background Radiations, proc. of IAU Symp. nr. 168, ed. by M. Kafatos, p. 183 
Giovanelli et al. 1996a, in preparation

Giovanelli et al. $1996 \mathrm{~b}$, in preparation

Lynden-Bell, D. Faber, S.M., Burstein, D., Davies, R.L., Dressler, A. Terlevick, R.J. \& Wegner, G. 1988, ApJ 326, 19

Mathewson, D.S., Ford, V.L. \& Buchhorn, M. 1992a ApJ 389, L5

Mathewson, D.S., Ford, V.L. \& Buchhorn, M. 1992b ApJS 81, 413

Piran, T., Lecar, M., Goldwirth, D., da Costa, L. \& Blumenthal, G. 1993, MNRAS 265, 681

Strauss, M. \& Willick, J. A. 1995, Physics Report 261, 271

Wegner, G., Colless, M., Baggley, G., Davies, R.L., Bertschinger, E., Burstein, D., McMahan Jr., R.K. \& Saglia, R.P. 1996, preprint.

Willick, J. A. 1990, ApJ 351, L5

Willick, J., Courteau, S., Faber, S. M., Burstein, D., Dekel, A. \& Kollat, T. 1996, ApJ, 457, 460

Willmer, C.N.A., da Costa, L.N., Pellegrini, P.S., Fairall, A. \& Latham, D. 1995, AJ 109, 61

Yahil, A. 1988, in Large-Scale Sructuture of the Universe,ed. V.C. Rubin G.V. Coyne (Princeton: Princeton University Press) p. 219 


\section{Figure Captions}

Fig. 1.- (Plate) - Density field along the Supergalactic plane reconstructed from the SFI sample. We show density contours in intervals of $\delta=0.2$, surface density maps with the height proportional to $\delta$ and density constrast maps. Bright yellow represents overdensities and dark blue underdense regions, respectively. See text or figure 2 for the identification of the structures.

Fig. 2.- Projected components of the three-dimensional peculiar velocity field along the Supergalactic plane superimposed to the density field. The contour spacing is 0.2 in $\delta$, with the heavy solid line indicating $\delta=0$. The coordinate system is as described in the text. The major structures seen in the map are the Great Attractor, the Perseus-Pisces complex, the Cetus region and the foreground of the Coma/A1367 supercluster. 


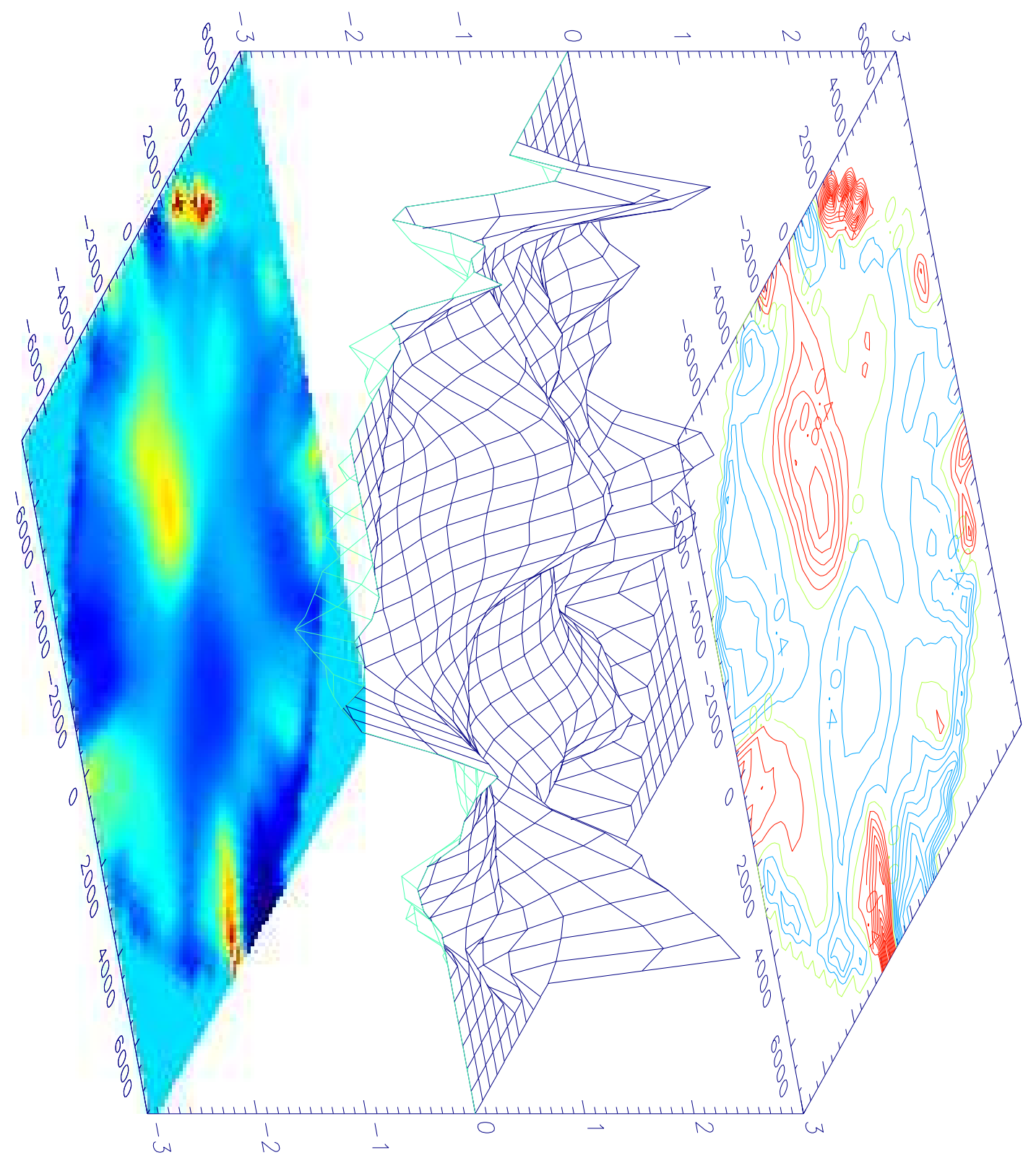




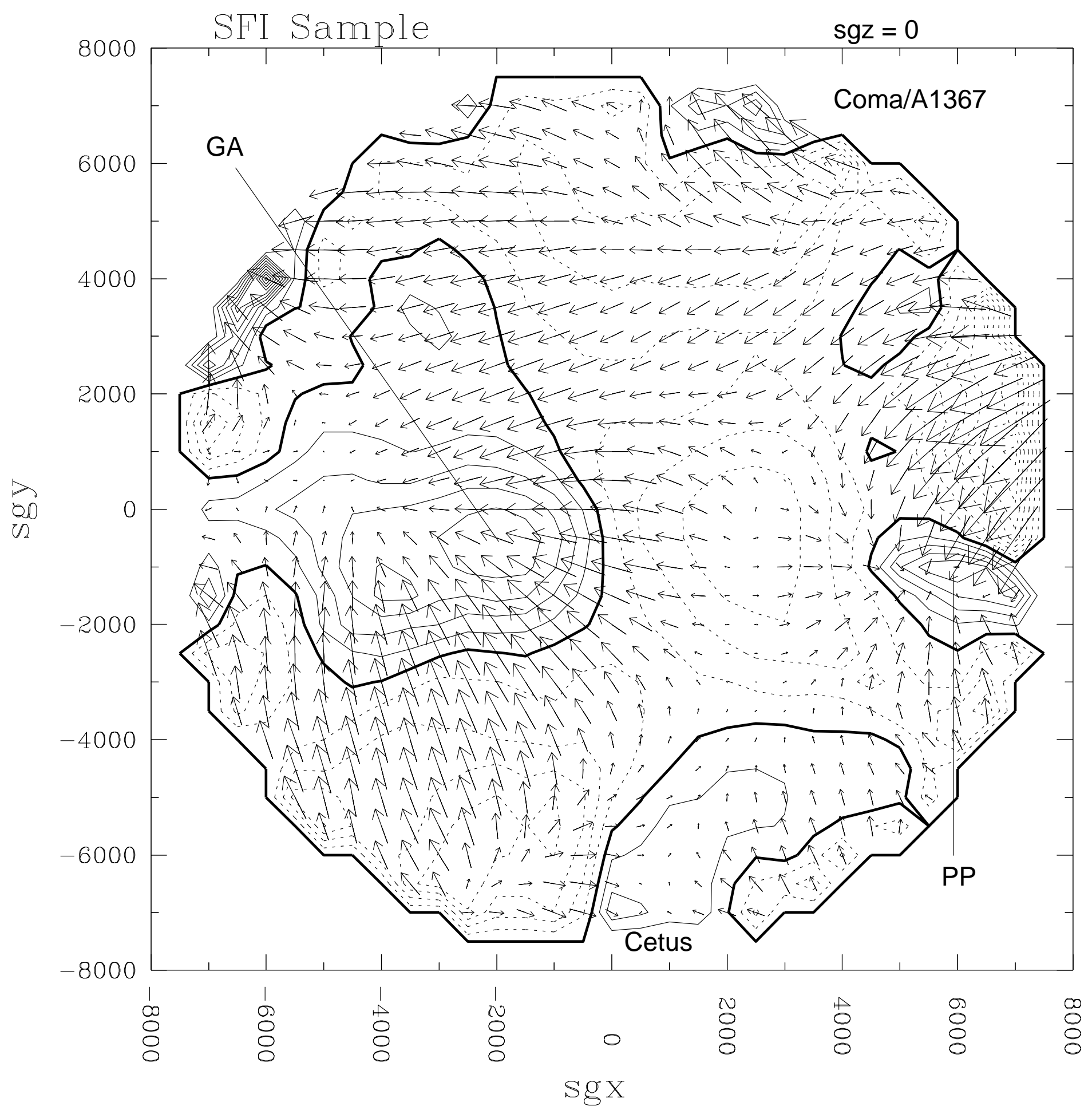

